\title{
Just Do It: Changing our Culture to Embrace Optimal Perioperative Venous Thromboembolism Chemoprophylaxis for Abdominal and Pelvic Cancer Patients
}

\author{
Srinivas J. Ivatury, MD, MHA ${ }^{1,2,3,4}$ and Stefan D. Holubar, MD, MS, FACS, FASCRS ${ }^{1,2,3,4}$ \\ ${ }^{1}$ Division of Colon \& Rectal Surgery, Dartmouth-Hitchcock Medical Center, Lebanon, NH; ${ }^{2}$ Geisel School of Medicine at \\ Dartmouth, Hanover, $\mathrm{NH} ;{ }^{3}$ Norris Cotton Cancer Center, Lebanon, $\mathrm{NH} ;{ }^{4} \mathrm{The}$ Dartmouth Institute for Health Policy \& \\ Clinical Practice, Lebanon, $\mathrm{NH}$
}

Early in the 21st century, postoperative venous thromboembolism (VTE), deep vein thrombosis (DVT), and pulmonary embolism (PE) continue to plague roughly 2.5$10 \%$ of abdominopelvic cancer patients. Cancer patients are an especially high-risk group, and VTE is the most common cause of early postoperative mortality, accounting for half of all deaths within 30 days after abdominopelvic cancer surgery.

Given that half of all VTE events occur after hospital discharge, there has been long-standing interest in extended chemoprophylaxis (ECP), typically for 28 days postoperatively. The hypothesis that ECP is more efficacious in preventing VTE than traditional hospital-only based prophylaxis has proven true; ECP also has been proven safe. Both randomized and large observation studies have shown that ECP after abdominopelvic cancer surgery significantly decreases VTE events but does not significantly increasing the risk of bleeding. ${ }^{1-4}$ Moreover, major organizations, such as the American Society of Chest Physicians (ACCP), American Society of Clinical Oncology (ASCO), and the National Comprehensive Cancer Network (NCCN), have published practice guidelines that recommend ECP. ${ }^{5-7}$ Despite this unified expert consensus regarding the benefit of ECP nationally, these recommendations have not been embraced and adoption of ECP has been poor. For example, Merkow et al. examined the adherence to

(C) Society of Surgical Oncology 2016

First Received: 25 January 2016; Published Online: 24 February 2016

S. D. Holubar, MD, MS, FACS, FASCRS e-mail: Stefan.D.Holubar@hitchcock.org;

stefan.holubar@dartmouth.edu postdischarge VTE prophylaxis in Medicare beneficiaries undergoing colorectal cancer surgery and found a compliance rate of a paltry $1.5 \%$ overall. $^{8}$

In this issue of Annals of Surgical Oncology, we are presented with two studies examining issues integral to the implementation of ECP into clinical practice. The first, by Fagarasanu et al., is a meta-analysis reexamining the efficacy and potential risk of ECP and represents the highestquality level of evidence supporting ECP to date. The second, by Krell et al., examines the operationalization of ECP using a Michigan statewide registry, highlighting that ECP has not been widely adopted.

Fagarasanu et al. reported the results of a well-performed systematic review and meta-analysis examining the efficacy of ECP. The authors, not surprisingly, found a significant reduction of proximal DVT, and all VTE, with ECP, with no increased rate of major bleeding. The three randomized control trials referenced in the Fagarasanu et al. meta-analysis are summarized below. The ENOXACAN-II study, by Bergqvist et al., randomized patients to ECP with low molecular weight heparin (LMWH), enoxaparin, or standard duration LMWH prophylaxis and found a lower rate of VTE in the ECP group of 4.8 versus $12.0 \%$ in controls. ${ }^{2}$ Of note, there was persistence of the protective effect at 3 months. In the European multinational CANBESURE study by Kakkar et al., patients were randomized to ECP with bemiparin, an ultraLMWH associated with a lower risk of bleeding than LMWH, or standard duration bemiparin prophylaxis, and similarly found a lower rate of major VTE in the ECP group of 0.8 versus $4.6 \%$ in controls; however, there was no difference in a composite outcome of DVT, nonfatal PE, and all-cause mortality. ${ }^{3}$ Finally, in Vedovati et al., patients 
who had laparoscopic colorectal cancer surgery were randomized to ECP with LMWH or discontinuation of LMWH following normal complete lower extremity compression ultrasonography on postoperative day eight. ${ }^{4}$ They observed a significantly lower rate of VTE in the ECP group, 0 versus $9.4 \%$ in controls, causing the study to be stopped on interim safety analysis. With the addition of the Fagarasanu et al. analysis to the already-present volume of literature supporting ECP for patients with abdominopelvic cancer, we advocate that the case regarding the clinical merit for ECP be closed. Future efforts on this topic should focus not on efficacy or safety, but on quality improvement, implementation, adoption, and policy-making.

Also in this issue, Krell et al., used the Michigan Surgical Quality Collaborative (MSQC) to shed light on the degree to which the evidence and guidelines have, or have not, penetrated surgical practices by describing VTE prophylaxis variation in Michigan. In their study, only $40 \%$ of patients received perioperative chemoprophylaxis (preoperative or intraoperative). Also, there was wide variation in the rate of overall use of prophylaxis ranging from $1.0 \%$ in the lowest quintile to $72.2 \%$ in the highest quintile, mostly driven by the variation in preoperative prophylaxis. In examining contributing barriers to the observed low rates of prophylaxis, the authors found a multifaceted set of reasons including lack of standardized system, physician beliefs, and lack of utilization of available risk assessment tools. Suprisingly, $74 \%$ of survey respondents state that their respective hospitals use electronic decision support to aid in VTE risk assessment. Despite this, there is poor compliance with even a single dose of preoperative chemoprophylaxis. Certainly, the findings in this study are echoed in hospitals throughout the United States. The next steps will need to include a collaborative approach to overcome the barriers of education and lenient systems.

Culture can be changed and outcomes can be improved. At Dartmouth-Hitchcock within our colorectal practice, we were able to achieve a postoperative VTE rate of just $0.84 \%$ versus the expected risk-adjusted rate of $2.3 \%$ as measured by the National Surgical Quality Improvement Project (NSQIP). This was done by instituting routine first dose of chemoprophylaxis before incision and routine ECP for both major abdominopelvic cancer and inflammatory bowel disease patients, which comprise $>80 \%$ of our abdominopelvic cases. Others have had similar experience. In a large retrospective study of $>5000$ patients from Memorial Sloan-Kettering cancer center, Selby et al. showed that giving the first dose of chemoprophylaxis preoperatively did not increase intraoperative bleeding or transfusion rates, and reduced the DVT rate from 1.3 to $0.2 \%$, and PE from 1 to $0.4 \% .^{9}$ Examples of optimal perioperative VTE prophylaxis such as these represent a fulfillment the promise of quality improvement: that hospitals and perioperative physicians can both measure outcomes, and "move the needle" and improve outcomes as well.

Optimal VTE prophylaxis, namely preoperative chemoprophylaxis and ECP, are efficacious, safe best practices, are the national standard of care, and must be embraced by hospitals, physicians, and policy makers. Clearly, it is time to "just do it." Optimal perioperative VTE prophylaxis will only be achieved by ongoing educational and quality improvement efforts to effect a change in our culture. We need to embrace optimal VTE prophylaxis for every patient. Only then can we give our patients the "best shot" at preventing of a major cause of avoidable morbidity and mortality.

\section{REFERENCES}

1. ENOXACAN Study Group. Efficacy and safety of enoxaparin versus unfractionated heparin for prevention of deep vein thrombosis in elective cancer surgery: a double-blind randomized multicentre trial with venographic assessment. $\mathrm{Br} J$ Surg. 1997;84(8):1099-103. (see appendix).

2. Bergqvist D, Agnelli G, Cohen AT, et al. Duration of prophylaxis against venous thromboembolism with enoxaparin after surgery for cancer. N Engl J Med. 2002;346(13):975-80.

3. Kakkar VV, Balibrea JL, Martínez-González J, et al. Extended prophylaxis with bemiparin for the prevention of venous thromboembolism after abdominal or pelvic surgery for cancer: the CANBESURE randomized study. J Thromb Haemost. 2010; 8(6):1223-9.

4. Vedovati MC, Becattini C, Rondelli F, et al. A randomized study on 1-week versus 4-week prophylaxis for venous thromboembolism after laparoscopic surgery for colorectal cancer. Ann Surg. 2014;259(4):665-9.

5. Gould MK, Garcia DA, Wren SM, et al. Prevention of VTE in nonorthopedic surgical patients: antithrombotic therapy and prevention of thrombosis, 9th edn. American College of Chest Physicians evidence-based clinical practice guidelines. Chest. 2012;141(2_suppl):e227S-e77S.

6. Lyman GH, Bohlke K, Khorana AA, et al. Venous thromboembolism prophylaxis and treatment in patients with cancer: American Society of Clinical Oncology Clinical Practice Guideline Update 2014. J Clin Oncol. 2015;33(6):654-6.

7. Khorana AA. The NCCN Clinical practice guidelines on venous thromboembolic disease: strategies for improving VTE prophylaxis in hospitalized cancer patients. Oncologist. 2007;12(11): 1361-70.

8. Merkow RP, Bilimoria KY, Sohn M-W, et al. Adherence with postdischarge venous thromboembolism chemoprophylaxis recommendations after colorectal cancer surgery among elderly Medicare beneficiaries. Ann Surg. 2014;260(1):103-8.

9. Selby LV, Sovel M, Sjoberg DD et al. Preoperative chemoprophylaxis is safe in major oncology operations and effective at preventing venous thromboembolism. J Am Coll Surg. 2015. doi:10.1016/j.jamcollsurg.2015.11.011. 